\title{
The anatomic rationale for transforaminal endoscopic interbody fusion: a cadaveric analysis
}

\author{
Mitchell Hardenbrook, MD, ${ }^{1}$ Sergio Lombardo, DO, ${ }^{2}$ Miles C. Wilson, BA, MBA, ${ }^{3}$ and \\ Albert $\mathrm{E}$. Telfeian, $\mathrm{MD}, \mathrm{PhD}^{4}$ \\ ${ }^{1}$ Advance Spine Institute, Boston; ${ }^{2}$ United States Naval Health Clinic, Annapolis, Maryland; ${ }^{3}$ A2 Medical, Boston, Massachusetts; \\ and ${ }^{4}$ Department of Neurosurgery, Rhode Island Hospital, The Warren Alpert Medical School of Brown University, Providence, \\ Rhode Island
}

OBJECTIVE The authors describe a cadaveric analysis to determine the ideal dimensions and trajectory for considering endoscopic transforaminal interbody implantation.

METHODS The soft tissues of 8 human cadavers were removed from L-1 to the sacrum, exposing the posterior bony elements. Facetectomies were performed bilaterally at each lumbar level with resection of the pars interarticularis, revealing the pedicles, nerve roots, and interbody disc space. Each level was digitally photographed with a marker for scale and evaluated with digital analysis software. The traversing and exiting nerve roots and pedicle margins were identified, and the distances between these structures and their relationships to the surrounding structures were documented.

RESULTS The dimensions of 2 areas were measured: the working triangle and safe zone. The working triangle is the triangle between the exiting and traversing nerve roots above the superior margin of the inferior pedicle. The safe zone is the trapezoid bounded by the widths of the superior and inferior pedicles between the exiting and traversing nerve roots. The mean surface area for the working triangle was $1.83 \mathrm{~cm}^{2}$, with L5-S1 having the largest area at $2.19 \mathrm{~cm}^{2}$. The mean surface area of the safe zone was $1.19 \mathrm{~cm}^{2}$, with L5-S1 having the largest area at $1.26 \mathrm{~cm}^{2}$. At the medial border of the pedicle extending superiorly, there were no nerve structures within $1.19 \mathrm{~cm}$ at any level. On the lateral border of the pedicle, the exiting nerve root was closer superiorly, with the closest being $0.3 \mathrm{~cm}$.

CONCLUSIONS The working triangle is a relatively large area. The safe zone, just superior to the pedicle, is free of nerve structures. By utilizing the superior border of the pedicle, the disc space can be accessed within this safe zone without risk of injury to the nerves. A thorough understanding of foraminal anatomy is fundamental for considering how to safely access the disc space, thereby utilizing less invasive endoscopic techniques, and is an important first step in considering what shapes and sizes of interbody implants and retractors are feasible for use in the foramen.

http://thejns.org/doi/abs/10.3171/2015.10.FOCUS15389

KEY WORDS endoscopic discectomy; minimally invasive; transforaminal; Kambin's triangle; TLIF; transforaminal lumbar interbody fusion

$\mathrm{I}$ N 1972, Kambin described a lumbar transforaminal discectomy via the posterolateral approach and defined Kambin's triangle as the site to approach the intervertebral disc. 2,3 Kambin's triangle is defined as a right triangle over the dorsolateral disc: the hypotenuse is the exiting nerve root, the base (width) is the superior border of the caudal vertebra, and the height is the traversing nerve root. ${ }^{2,3}$ Advances in endoscopic visualization and instrumentation, as well as increased patient demand for more minimally invasive procedures, have led to an increased popularity of the transforaminal endoscopic discectomy technique.
Transforaminal lumbar interbody fusion (TLIF) was first introduced by Harms and colleagues as an interbody fusion technique that provided the surgeon the ability to simultaneously decompress the neural elements with limited neural retraction and take advantage of the single approach in order to also achieve circumferential arthrodesis. $^{6,7}$ The minimally invasive TLIF procedure has been recently shown to reduce morbidity and health care costs in comparison with open TLIF. ${ }^{5,8,10,11,14} \mathrm{We}$ set out to evaluate the relevant anatomy at the neural foramen in consideration for the development of an interbody fusion device, appropriate instruments for neural decompression, and

ABBREVIATIONS TLIF = transforaminal lumbar interbody fusion 
endplate preparation that would be optimal in the evolution of the development of the minimally invasive TLIF procedure into a fully endoscopic version.

\section{Methods}

The soft tissues of 8 fresh-frozen human cadavers (4 males and 4 females with no history of previous spine surgery) were removed from L-1 to the sacrum, exposing the posterior bony elements. None of the specimens exhibited gross evidence of congenital or acquired spinal anomalies. Facetectomies were performed bilaterally at each lumbar level with resection of the pars interarticularis, revealing the pedicles, nerve roots, and interbody disc space. Each level was digitally photographed with a marker for scale and evaluated using digital analysis software (Scion Image). The traversing and exiting nerve roots and pedicle margins were identified, and the distances between these structures and their relationship to the surrounding structures were documented. A total of 80 foraminal levels were studied.

\section{Results}

The dimensions of 2 areas were measured: the working triangle and the safe zone. The working triangle is the triangle between the exiting and traversing nerve roots above the superior margin of the inferior pedicle (Fig. 1). The safe zone is the trapezoid bound by the widths of the superior and inferior pedicles between the exiting and traversing nerve roots (Fig. 2). The mean surface area for the working triangle was $1.83 \mathrm{~cm}^{2}$, with L5-S1 having the largest area at $2.19 \mathrm{~cm}^{2}$ (Fig. 3 and Table 1). The mean surface area for the safe zone was $1.19 \mathrm{~cm}^{2}$, with L5-S1 having the largest area at $1.26 \mathrm{~cm}^{2}$ (Fig. 3 and Table 1).

The hypotenuse of our foraminal working triangle is the traversing nerve root. For each lumbar level evaluated, we measured 2 distances: the distance to the traversing nerve from the pedicle at the medial pedicle wall, and the

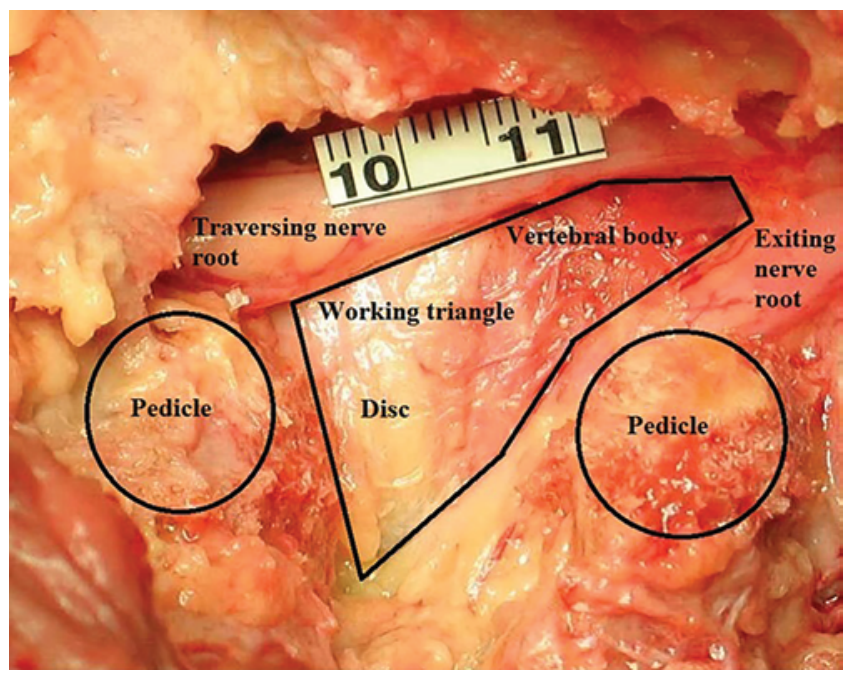

FIG. 1. Cadaveric demonstration of the working triangle in the L5-S1 neural foramen. The working triangle is the triangle between the exiting and traversing nerve roots above the superior margin of the inferior pedicle.

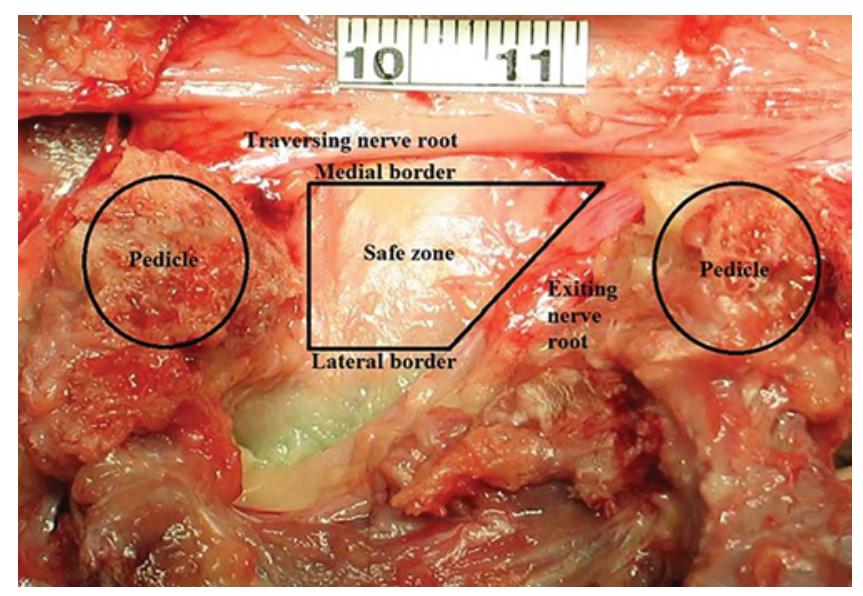

FIG. 2. Cadaveric demonstration of the safe zone in the L3-4 neural foramen. The safe zone is the trapezoid bounded by the widths of the superior and inferior pedicles between the exiting and traversing nerve roots.

distance to the traversing nerve from the pedicle at the lateral pedicle wall (Fig. 4 and Table 2). At the medial border of the pedicle extending superiorly, there were no nerve structures within $1.19 \mathrm{~cm}$ at any level. On the lateral border of the pedicle, the exiting nerve root was closer superiorly, with the closest being $0.39 \mathrm{~cm}$. The average distance to the traversing nerve from the pedicle was $1.79 \mathrm{~cm}$ at the medial wall and $1.00 \mathrm{~cm}$ at the lateral wall.

\section{Discussion}

The TLIF procedure takes advantage of an anatomical corridor that allows for both decompression of the exiting and traversing nerve roots and access to the interbody space in order to achieve simultaneous decompression and fusion. The potential advantages of converting a minimally invasive TLIF to a surgery performed under fully endoscopic visualization include a smaller incision, decreased blood loss, shorter patient recovery time, and the possibility of performing the surgery without general anesthesia. ${ }^{5,8,11-13}$

The significant anatomical feature of the lumbar neural foramen that makes endoscopic TLIF a challenge is the small working area between the traversing and exiting roots through which 1) a large amount of facet bone and disc material must be removed to achieve satisfactory nerve decompression, 2) sufficient endplate preparation

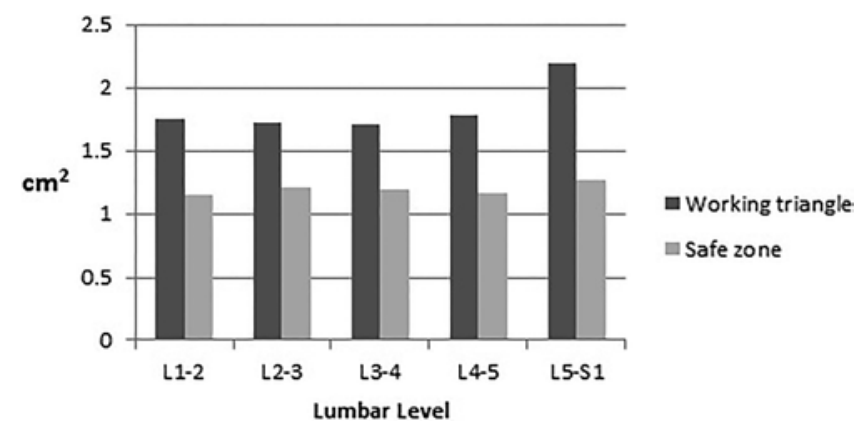

FIG. 3. Mean area $\left(\mathrm{cm}^{2}\right)$ of the working triangle and safe zone at each neural foramen calculated from 8 cadaveric specimens. 
TABLE 1. Mean area per lumbar level*

\begin{tabular}{ccc}
\hline Level & Working Triangle & Safe Zone \\
\hline L1-2 & $1.75(1.17-2.39)$ & $1.15(0.78-1.49)$ \\
\hline L2-3 & $1.72(0.88-2.36)$ & $1.2(0.70-1.57)$ \\
\hline L3-4 & $1.71(1.07-2.66)$ & $1.19(0.83-1.45)$ \\
\hline L4-5 & $1.78(1.02-2.07)$ & $1.16(0.85-1.65)$ \\
\hline L5-S1 & $2.19(1.34-3.71)$ & $1.26(0.98-1.85)$ \\
\hline
\end{tabular}

* Values are presented as $\mathrm{cm}^{2}$ (range).

must be performed, and 3) an interbody implant and fusion substrate must be inserted. Adequate neural decompression, sufficient endplate preparation, and interbody device delivery are the 3 most significant challenges in the evolution of the minimally invasive TLIF procedure to becoming an endoscopic procedure. The data presented here indicate that a safe zone area that is the width of the pedicle exists at the neural foramen and is about $1.2 \mathrm{~cm}^{2}$ in size. The average width of the space between the inferior pedicle and the exiting nerve is $1.79 \mathrm{~cm}$ at the medial border of the pedicle and $1 \mathrm{~cm}$ at the lateral border of the pedicle; these dimensions are in the realm of standard nonexpandable interbody cages.

Several earlier studies have looked at the detailed anatomy of the lumbar neural foramina with respect to determining the maximum working channel size suitable to performing endoscopic discectomy procedures. ${ }^{1,9,10,15}$ Mirkovic et al. described the dimensions of the safe margins for a working cannula in the neural foramen in 1995 and determined that a cannula placed in line with the medial one-third of the pedicle could sustain a safe average diameter of $7.5 \mathrm{~mm} .{ }^{10}$ Min et al. in 2005 determined the mean distance between the exiting nerve root and the superior articulating process to be $11.6 \mathrm{~mm}$, which is sufficiently smaller than our own determination of $15.5 \mathrm{~mm}$ for the average distance between the exiting and traversing nerve roots at the top of the base of the working triangle with the superior articulating process removed. ${ }^{9}$ These studies and earlier versions of the endoscopic discectomy procedure did not account for the possibility of the minimally invasive removal of the superior articulating process to expose the traversing nerve root. The data presented here provide measurements of the foraminal content dimensions with simulated foraminal bone removal.

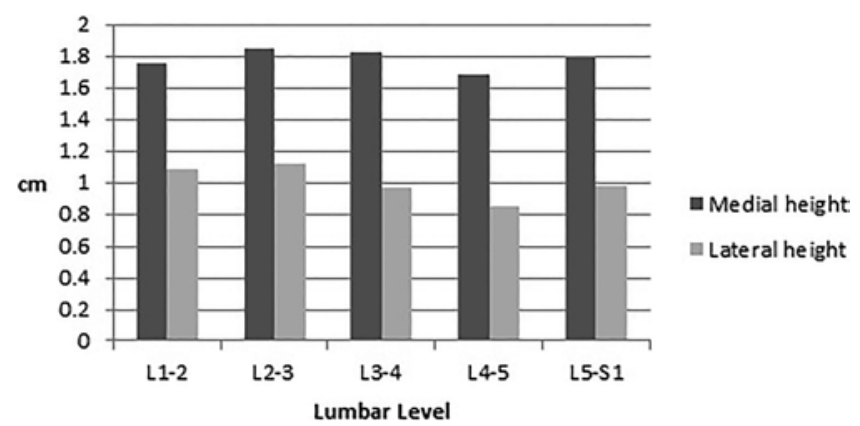

FIG. 4. Mean distance from the inferior pedicle (shown from the medial and lateral pedicle borders) to the exiting nerve root zone at each neural foramen calculated from 8 cadaveric specimens.
TABLE 2. Mean distance from pedicle*

\begin{tabular}{ccc}
\hline Level & Medial Height & Lateral Height \\
\hline L1-2 & $1.75(1.31-2.08)$ & $1.08(0.65-1.34)$ \\
\hline L2-3 & $1.85(1.39-2.43)$ & $1.12(0.8-1.53)$ \\
\hline L3-4 & $1.83(1.38-2.14)$ & $0.96(0.68-1.22)$ \\
\hline L4-5 & $1.68(1.2-2.2)$ & $0.85(0.61-1.23)$ \\
\hline L5-S1 & $1.79(1.45-2.46)$ & $1.77(1.12-2.22)$ \\
\hline
\end{tabular}

* Values are presented as $\mathrm{cm}$ (range).

Percutaneous endoscopic TLIF has been described previously in clinical studies ${ }^{4,16}$ and in a 3D surgical simulation and cadaveric experiment. ${ }^{13}$ In one clinical study, the authors admitted to a $36 \%$ complication rate and did not recommend the procedure unless "decisive technical improvement" was made. ${ }^{4}$ The anatomical data presented here describe the boundaries for future technical advancements in order to make minimally invasive endoscopic TLIF feasible, essentially opening the door for a dynamic solution to what was a static problem. Once the disc space is accessed safely at the safest point in the working zone, the necessary bone and disc removal for decompression and fusion preparation could be performed, as well as dynamic disc space distraction. Earlier versions of the endoscopic discectomy technique accessed the disc space in the center of the disc at about the middle of the pedicle and from a less lateral starting point. More modern endoscopic approaches access the inferior disc space-superior endplate junction at the medial wall of the pedicle. Once in the disc, we suggest the concept of shared circumferential distraction-retraction: incremental disc space dilation that achieves exponential $\left(\pi r^{2}\right)$ increases in the area achieved for interbody implant placement at the cost of incremental nerve root distraction.

One other unique feature of transforaminal endoscopic discectomy, as it applies to the endoscopic TLIF procedure, is the angle of access to the disc space. The typical starting point for transforaminal endoscopic discectomy is 10 to $16 \mathrm{~cm}$ off the midline; this allows the starting needle to enter the disc space from an oblique trajectory just sliding off the ventral portion of the superior articulating process and entering the disc space just underneath the traversing nerve root. This more oblique access to the disc could allow for adequate neural decompression with removal of less than $50 \%$ of the facet bone. The result opens up the possibility of a stand-alone interbody fusion because less facet bone is removed (Jacquot and Gastambide performed stand-alone endoscopic TLIF in $81 \%$ of their 57 patients studied and found no difference in the fusion rates between instrumented vs stand-alone cases). ${ }^{4}$

The data presented here represent only a cadaveric anatomical study on what are presumed to be normal neural foramina; this is not an endoscopic TLIF feasibility study. Several other points must be made before extrapolating these data to a clinical scenario: 1) the method used to remove the posterior bony elements in this study did not simulate performing this procedure endoscopically; 2) the preparation and preservation of the cadaver tissue used in a study like this, which looks at soft tissue in addition to bony and cartilaginous anatomy, can artificially impact 
the results; 3) the working and safe zone measurements presented here could vary widely in patients with spondylolisthesis and disc collapse, which are the typical pathologies treated with the TLIF procedure; and 4) the conjoined nerve roots or abnormal anatomy could also influence the ability to make generalizations based on this study.

\section{Conclusions}

The working triangle between the exiting and traversing nerves in the lumbar neural foramen is a relatively large area. The safe zone, just superior to the pedicle, is free of nerve structures. By targeting the superior medial border of the inferior pedicle, the disc space can be accessed within this safe zone without risk of injury to the nerves. A thorough understanding of the foraminal anatomy is fundamental for considering how to safely access the disc space utilizing less invasive endoscopic techniques and is an important first step in considering what shapes and sizes of interbody implants and retractors are feasible for use in the foramen.

\section{References}

1. Arslan M, Cömert A, Açar Hİ, Ozdemir M, Elhan A, Tekdemir I, et al: Nerve root to lumbar disc relationships at the intervertebral foramen from a surgical viewpoint: an anatomical study. Clin Anat 25:218-223, 2012

2. Harms JG, Jeszenszky D: The unilateral transforaminal approach for posterior lumbar interbody fusion. Orthop Traumatol 6:88-99, 1998

3. Harms J, Rolinger H: [A one-stage procedure in operative treatment of spondylolistheses: dorsal traction-reposition and anterior fusion.] Z Orthop Ihre Grenzgeb 120:343-347, 1982 (Ger)

4. Jacquot F, Gastambide D: Percutaneous endoscopic transforaminal lumbar interbody fusion: is it worth it? Int Orthop 37:1507-1510, 2013

5. Lee JC, Jang HD, Shin BJ: Learning curve and clinical outcomes of minimally invasive transforaminal lumbar interbody fusion: our experience in 86 consecutive cases. Spine (Phila Pa 1976) 37:1548-1557, 2012

6. Kambin P: Arthroscopic microdiscectomy. Spine J 3 (3 Suppl):60S-64S, 2003

7. Kambin P, Sampson S: Posterolateral percutaneous suctionexcision of herniated lumbar intervertebral discs. Report of interim results. Clin Orthop Relat Res (207):37-43, 1986

8. McGirt MJ, Parker SL, Lerner J, Engelhart L, Knight T, Wang MY: Comparative analysis of perioperative surgical site infection after minimally invasive versus open posterior/ transforaminal lumbar interbody fusion: analysis of hospital billing and discharge data from 5170 patients. J Neurosurg Spine 14:771-778, 2011
9. Min JH, Kang SH, Lee JB, Cho TH, Suh JK, Rhyu IJ: Morphometric analysis of the working zone for endoscopic lumbar discectomy. J Spinal Disord Tech 18:132-135, 2005

10. Mirkovic SR, Schwartz DG, Glazier KD: Anatomic considerations in lumbar posterolateral percutaneous procedures. Spine (Phila Pa 1976) 20:1965-1971, 1995

11. Phan K, Rao PJ, Kam AC, Mobbs RJ: Minimally invasive versus open transforaminal lumbar interbody fusion for treatment of degenerative lumbar disease: systematic review and meta-analysis. Eur Spine J 24:1017-1030, 2015

12. Shunwu F, Xing Z, Fengdong Z, Xiangqian F: Minimally invasive transforaminal lumbar interbody fusion for the treatment of degenerative lumbar diseases. Spine (Phila Pa 1976) 35:1615-1620, 2010

13. Wang J, Zhou Y, Zhang ZF, Li CQ, Zheng WJ, Liu J: Comparison of one-level minimally invasive and open transforaminal lumbar interbody fusion in degenerative and isthmic spondylolisthesis grades 1 and 2. Eur Spine J 19:1780-1784, 2010

14. Wang Y, Le DQ, Li H, Wang M, Bünger CE: Navigated percutaneous lumbosacral interbody fusion: a feasibility study with three-dimensional surgical simulation and cadaveric experiment. Spine (Phila Pa 1976) 36:E1105-E1111, 2011

15. Xin G, Shi-Sheng H, Hai-Long Z: Morphometric analysis of the YESS and TESSYS techniques of percutaneous transforaminal endoscopic lumbar discectomy. Clin Anat 26:728734, 2013

16. Yao N, Wang W, Liu Y: Percutaneous endoscopic lumbar discectomy and interbody fusion with B-Twin expandable spinal spacer. Arch Orthop Trauma Surg 131:791-796, 2011

\section{Disclosures}

The authors report no conflict of interest concerning the materials or methods used in this study or the findings specified in this paper.

\section{Author Contributions}

Conception and design: Telfeian, Hardenbrook, Lombardo. Acquisition of data: Telfeian, Hardenbrook, Lombardo. Analysis and interpretation of data: all authors. Drafting the article: all authors. Critically revising the article: all authors. Reviewed submitted version of manuscript: all authors. Statistical analysis: all authors. Administrative/technical/material support: Telfeian, Hardenbrook, Wilson. Study supervision: Telfeian, Hardenbrook, Lombardo.

\section{Correspondence}

Albert E. Telfeian, Department of Neurosurgery, Rhode Island Hospital, 593 Eddy St., Providence, RI 02903. email: atelfeian@ lifespan.org. 